\title{
Trajectory forms as a source of information about events
}

\author{
MICHAEL M. MUCHISKY and GEOFFREY P. BINGHAM \\ Indiana University, Bloomington, Indiana
}

\begin{abstract}
The ability to use trajectory forms as visual information about events was tested. A trajectory form is defined as the variation in velocity along a path of motion. In Experiment 1, we tested the ability to detect trajectory form differences between simulations of a freely swinging pendulum and a handmoved pendulum. The trajectory form of the freely swinging pendulum was symmetric around the midpoint, whereas the hand moved was not. In Experiment 2, we isolated trajectory form information by varying the amplitudes of events while holding their periods constant. Straight path versions of the harmonic events from Experiment 1 were tested. In Experiment 3, we tested sensitivity to symmetrical peakening or flattening of trajectory forms. Participants detected small differences in all three experiments. In Experiment 4, we tested the ability to identify specific events based only on differences in trajectory forms. Participants were able to identify four different events. We investigated properties of trajectory forms that might potentially be detected and used as information, and we found that the curvature yielded good results.
\end{abstract}

In conditions that isolate motion as visual information, human observers have been shown to be able to distinguish and identify a large number of different events (see Bingham, 1995, Jansson, Bergström, \& Epstein, 1994, and Johansson, von Hofsten, \& Jansson, 1980, for reviews). How does structure in motion provide information about events? Johansson $(1950,1973,1976)$ suggested that patterns of relative motion in optical flow yield apprehension of specific types of events. For instance, a display consisting of two dots traveling on the rim and hub of an unseen rolling wheel is perceived as a rolling wheel, but not when one of the two dots is removed. These and related early observations led to the now burgeoning field of structure-from-motion studies (see, e.g., Braunstein, 1976, 1994, Lappin, 1995, Todd, 1995, and Todd, Tittle, \& Norman, 1995, for reviews). In these studies, the ability to perceive three-dimensional shape using motion-generated information was investigated, along with the ability to discriminate, for instance, whether motions are rigid or nonrigid. The optic flow information contained in structure-from-motion displays is most often analyzed in terms of the structure available from only two or three successive frames of a display (see Norman \& Todd, 1993, Perotti, Todd, \& Norman, 1996, and Todd, 1982, 1983, for notable exceptions). As indicated by the temporally local nature of this information, these are studies of object perception in the context of events, rather than of event recognition.

Events are inherently time extended and concern the form of an object's motion, not just the form of the ob-

Portions of this manuscript were used in partial fulfillment of the requirements of a $\mathrm{PhD}$ for M.M.M. Correspondence should be addressed to G. P. Bingham, Department of Psychology, 10th and Grove Streets, Indiana University, Bloomington, IN 47405 (e-mail: gbingham@indiana.edu). ject. For example, Johansson (1973) discovered that human walking and other activities could be recognized when the point-light technique was used to isolate the motions of a moving person in a display. Temporally local optic flow analyses have been applied to the perception of the rigid link-segment structure of a person in such a display (Hoffman \& Flinchbaugh, 1982), but this link-segment structure can be seen in the context of different eventsfor instance, walking versus running or dancing. These varieties of locomotion can also be recognized, but the structure-from-motion analysis does not address this. As argued by Bingham (1995), two- or three-frame analysis is insufficient to reveal information in optic flow for event recognition. For instance, Johansson (1976) found that point-light displays of human walking in which the higher frequencies of motion had been removed via Fourier analysis were no longer recognized as normal human walking. Rather, the walkers appeared to float along without actually walking. Observers described the forcefulness of the motion as absent. Of course, walking would also not be seen without the (low-frequency) fundamental. Such frequency components cannot be meaningfully considered in two- or three-frame displays. Clearly, more temporally extended forms of information must be involved in the ability to distinguish and recognize events.

Runeson (1974) provided some evidence that observers can distinguish and recognize events involving specific variations in velocity along a path of motion. In his study, observers viewed displays of a circle moving along a straight path across a screen with various motion functions, including constant velocity, constant acceleration, constant deceleration, and acceleration to a constant velocity. The mean velocity was the same in each case. After viewing each display, observers were asked to draw the 
motion as graphs of the velocity at each position on the screen (i.e., phase portraits). When reporting his results, Runeson focused on the fact that the observers' graphs did not accurately represent the motions shown. However, he also reported that observers drew different portraits for each of the different motion functions. This indicated that observers could distinguish the forms of motion. In addition, each observer saw and described each motion function more than once, and they drew the same phase portraits for each motion function each time they viewed it, indicating that they could also recognize the particular motions. These results were replicated by Bingham and Runeson (1983).

Bingham (1995) subsequently suggested that trajectory forms might provide information for event recognition. Trajectory form was defined to include both the shape of the path of motion and the pattern of variation in speed along the path of motion. A trajectory form remains invariant over changes in those aspects of an optical trajectory that vary with viewing distance or sizes in an event - that is, changes in amplitude or frequency. One is able to recognize a swinging pendulum, a walking person, or a limping person whether each is viewed nearby or farther away. A change in the viewing distance changes the optical amplitude of an event, but not the form or the observer's ability to recognize the event. Furthermore, both people and pendulums can be recognized for what they are, despite variations in their sizes. Pendulums of different lengths will vary in their frequency of swinging, and people of different heights will vary in their frequency of stepping, but neither will vary in their essential form of motion. The trajectory form that is common to these situations can be captured in a dimensionless phase portrait - that is, a plot of position versus velocity, normalized using the amplitude and frequency.

Bingham, Rosenblum, and Schmidt (1995) investigated the perception of a variety of different types of events, including inanimate and animate events that varied only in trajectory form. Inanimate events were created by filming patch-light displays of simple physical events, such as a freely swinging pendulum or a compression spring free-falling and bouncing. Animate events were produced by manually controlling the motions of the pendulum or spring to imitate the inanimate displays. The period of each manual event was matched to the corresponding inanimate event by means of a metronome set to the period of the inanimate event. Similarly, the amplitudes of the manual events were matched to the respective inanimate events by placing marks on the video screen at the endpoints of movement and using the marks to guide the actor. Actors moved the pendulum or spring by hand between the specified endpoints of motion in time to the metronome. However, while both amplitude and period were kept constant, the trajectory form was free to vary.

In the inanimate pendulum event, the phase portrait spiraled inward toward a point representing the pendulum at rest - that is, with the rod hanging vertically. Amplitude decreased continuously due to the friction at the hinge. As shown in the top of Figure 1A, this produced a trajectory form in which the peak velocity always occurred at the middle of the swing. In the manually controlled pendulum, the actor failed to move exactly like the inanimate pendulum. Producing the requisite frequency while progressively reducing the amplitude was difficult to do. The result was an asymmetric trajectory form in which the trajectory crossed itself. The peak velocity of the manually controlled pendulum did not occur reliably at the middle of the swing. A shift in the location of the peak velocity between swings produced the crossing of trajectories in the phase portrait shown in the bottom of Figure 1B. For the freely moving and hand-moved springs, the periods and amplitudes were identical. Only the shape of the trajectory was different.

Observers in Bingham et al.'s (1995) study could distinguish the freely moving events and hand-moved events, despite identical periods and amplitudes. Because the periods and amplitudes were the same, Bingham et al. argued that variations in the trajectory forms provided visual information enabling the observers to distinguish these events.

If observers are in fact able to distinguish and recognize events based on differences in trajectory forms, then what types of differences are they able to detect? Meaningful types of variations to investigate would be those generated by variations in the underlying dynamics. For instance, in the Bingham et al. (1995) study, observers distinguished inanimate motions from animate motions generated by human limb movements. The latter type of motions has been successfully modeled using nonlinear damping and spring elements in dynamical models. Kay, Saltzman, and Kelso (1991) modeled rhythmic limb movements using a combination of Rayleigh and van der Pol nonlinear damping. Adding a nonlinear damping component to a harmonic oscillator can produce a skewing of the original circular trajectory form (Thompson \& Stewart, 1986). Can observers detect such asymmetry in a trajectory form? Alternatively, adding a nonlinear spring term (i.e., a hard or soft spring) can produce changes in the kurtosis of the harmonic form. Can observers detect, for instance, a change from a circular to a rounded rectangular or rounded diamond shape? Unlike the changes generated by the nonlinear damping, these changes preserve the symmetry of the original harmonic form. We investigated these possibilities.

\section{EXPERIMENT 1 Asymmetry in the Trajectory Forms of Simulated Pendulum Movements}

In Bingham et al. (1995), trajectory forms were generated by filming actual inanimate and manual events. However, these forms could not be varied parametrically. In the present experiment, oscillatory events were created using dynamical models to produce a range of trajectory forms from ones identical to the inanimate events of Bingham et al.'s study to those similar to human movements. The use of simulations allowed parametric 


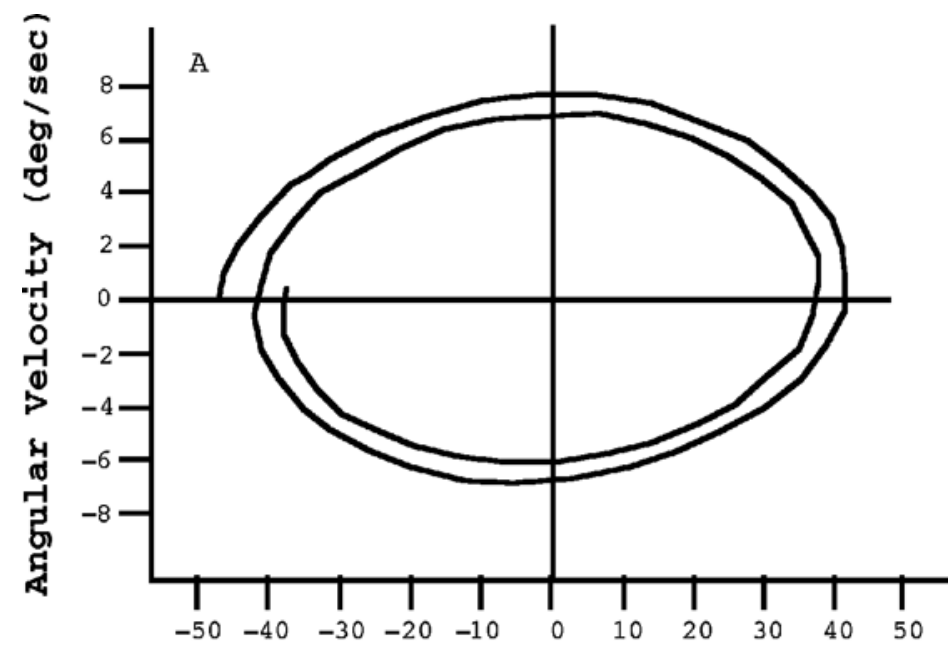

Angular Position (deg)

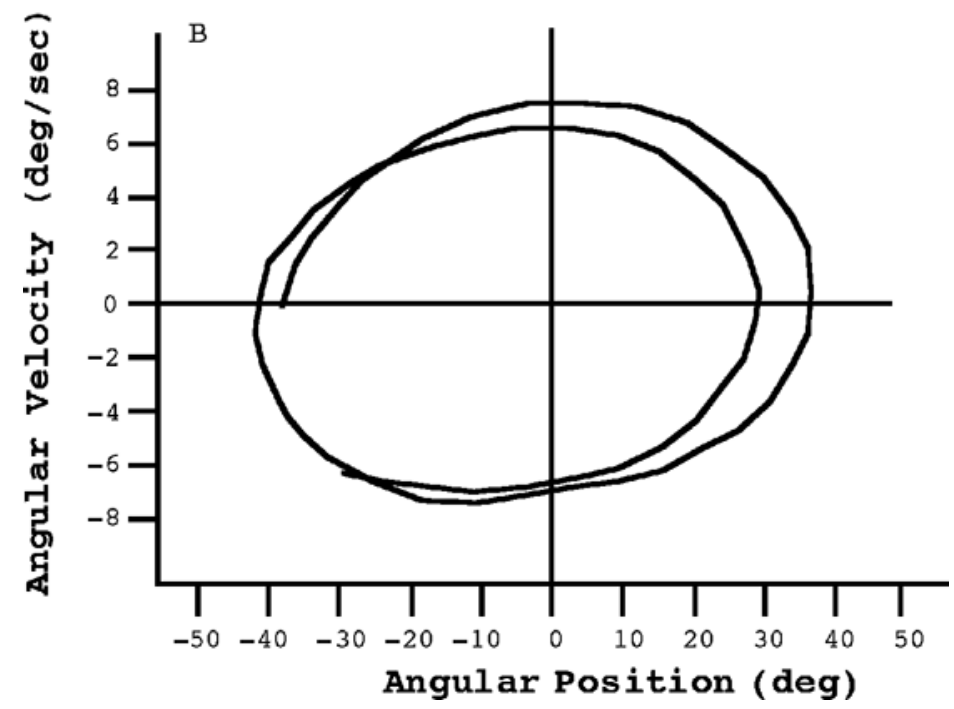

Figure 1. (A) Freely moving pendulum trajectory form from Bingham, Rosenblum, and Schmidt (1995). (B) Hand-moved pendulum trajectory form from Bingham et al. (1995).

variation of trajectory forms while controlling the amplitude and frequency of the events.

\section{Method}

Participan ts. An author (G.B.) and a computer programmer (M.S.) from the Indiana University Psychology Department participated in this experiment. Their ages were 38 and 28 years old. The computer programmer was paid $\$ 5$ per hour for his participation. Both had normal or corrected-to-normal vision.

Display generation. Two dynamic models were used to capture the qualitative nature of the two pendular events from Bingham et al. (1995): the symmetric freely swinging pendulum and the asymmetric hand-moved pendulum. A symmetric trajectory form was produced by simulating a gravitational force acting on a ball constrained to move along an arc by a rod on a frictionless hinge. The equation of motion was

$$
\ddot{\theta}=\frac{-\mathrm{g}}{l} \sin \theta,
$$

where 1 is the length of the rod, $g$ is the gravitational constant, and $\theta$ is the angle of the rod with the vertical. The rod was not visible in the display. The participants saw a black circle swing along a curved arc side to side on a white background. The standard displays were generated by setting $g / 1$ to 9.93 . The asymmetric shape of the trajectory form of the hand-moved pendulum was produced using a van der Pol oscillator:

$$
\ddot{\theta}=\lambda \dot{\theta}\left(\theta^{2}-1\right)-k \theta,
$$

where $\theta$ is the angular position of the pendulum, $k$ is a stiffness constant, and $\lambda$ is a positive coefficient on a nonlinear damping term. The van der Pol oscillator allowed us to vary the trajectory form from that of a symmetric and elliptical form characteristic of the freely moving pendulum to the asymmetric trajectory form characteristic of the hand-moved pendulum, as shown in Figure 2. The asymmetry produced by the dissipative term in the van der Pol dynamic results in a shift in the location of the peak velocity away from the midpoint of the swing, where the pendulum is vertical $(\theta=$ 


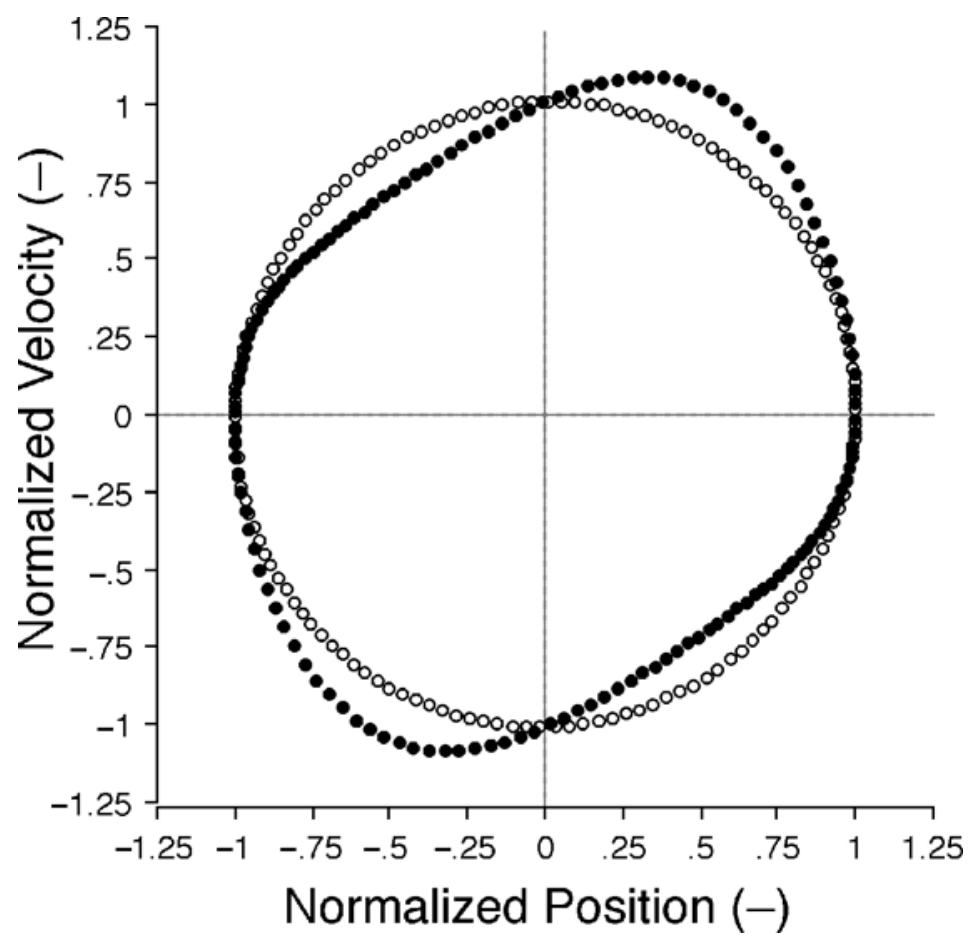

Figure 2. Trajectory form of model of hand-moved pendulum (van der Pol) (filled circles) and model of freely moving pendulum (harmonic) (unfilled circles). Trajectory forms in this figure and this experiment were isolated for analysis by normalizing the phase space using the frequency and amplitude of the motion. The result was a dimensionless form that shows the trajectory form. Position values were divided by amplitude, and velocity values were divided by the quantity ( $2 \pi *$ amplitude $*$ frequency).

$\left.0^{\circ}\right)$. To produce three levels of perturbation, $\lambda$ was set to values of $.15, .45$, or .90 , and $k$ was set to 8.70 . One difference between the hand-moved pendulum and this simulation was that the crossing of trajectories was not duplicated. Simulation of crossing trajectories would require time-dependent changes in both the dynamics and the resulting trajectory form (i.e., skewing the form in opposite directions). Our present goal was to test the participants' abilities to use trajectory forms as information about events. Sensitivity to time-dependent changes in trajectories as potential information about animacy is a separate issue.

The two pendular events were simulated to occur in the frontoparallel plane. The displays were generated using a software package called Interactive Physics II, run on a Macintosh IIci. Displays were presented on a 12 -in. monitor with a screen resolution of $640 \times 420$ pixels per inch and a refresh rate of $66.67 \mathrm{~Hz}$. A fourth-order Runga-Kutta algorithm was used to numerically integrate the equations of motion to yield the positions of the pendulum at successive time steps. A time step of $0.015 \mathrm{sec}$ was used, yielding a frame rate of $66.67 \mathrm{~Hz}$ to coincide with the refresh rate of the computer monitor. The frame rate of the displays was synchronized to that of the monitor by an acceleration/compression process in MacroMind Accelerator. Display presentation was controlled by the multimedia program MacroMind Director 3.1.

Pairs of displays containing simulations of the hand-moved pendulum and the freely swinging pendulum displays were always of the same amplitude and period. The amplitude was $36^{\circ}$ of swing. The period was $2.055 \mathrm{sec}$. The participants sat approximately $65 \mathrm{~cm}$ from the monitor, so that the total horizontal displacement of motion of the circle at the end of the pendulum subtended a visual angle of $4.49^{\circ}$. The average visual velocities were, respectively, $4.37^{\circ}$ or $-4.37^{\circ}$ per second for the first and second halves of the event. We used a frequency to which the visual system is likely to be well attuned - that characteristic of human movement. Also, these velocities were within the range often studied in the motion perception literature (McKee, 1981; Orban, de Wolf, \& Maes, 1984; Werkhoven, Snippe, \& Toet, 1992).

Procedure. The participants performed a two-alternative forcedchoice (2AFC) task in which one display was the freely swinging pendulum (the "standard" pendulum) and the other a hand-moved pendulum (the "perturbed" pendulum). Displays were presented successively. The participants' task was to indicate which was the perturbed pendulum in each pair of displays. They recorded their judgments on a protocol sheet. Three levels of perturbation, along with catch trials, were tested in 1- to 1.5-h sessions of 8-12 blocks per session over a 4-day period, for a total of 693 trials per participant. Each block contained 21 trials in a completely random order, including three repetitions of each of the seven basic event combinations of perturbed (P1-3) and standard (Std) events (P3-Std, P2-Std, P1-Std, Std-Std [catch trial], Std-P1, Std-P2, Std-P3). The purpose of the catch trials was to assess potential biases in judgments.

Neither head nor eye motion was constrained. We investigated whether the participants could detect trajectory form differences under conditions representative of normal viewing. Participants might detect trajectory form differences using either patterns of motion on the retina (see Werkhoven et al., 1992) or eye movements. These alternatives were not investigated or controlled.

At the beginning of each experimental session, the participants were given a short demonstration of the differences between the standard and perturbed displays using the strongest level of pertur- 

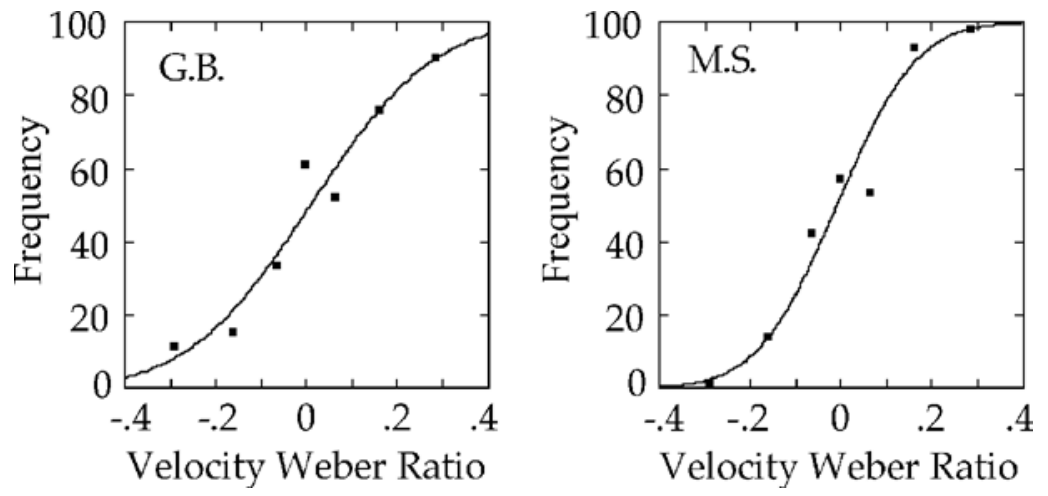

Figure 3. Cumulative normal curves fitted by probit analysis to the observed frequencies in selecting the second display as distorted. Curves and data plotted against mean velocity Weber ratios for Experiment 1.

bation to be shown in the experimental trials. The participants participated in three sample trials. No feedback was provided during the demonstration or the experimental trials.

\section{Results}

The frequency of responses identifying the second display as containing the perturbed pendulum was the dependent variable. This frequency was determined for each level of perturbation in each of the two orderings: perturbed pendulum first and perturbed pendulum second.

Using normalized trajectories, we quantified the amount by which the trajectory form was perturbed in terms of the average of the velocity Weber fractions calculated between the perturbed and standard events at equivalent time steps. ${ }^{1}$ Specifically, the perturbation measure was computed as follows:

$$
\frac{\sum_{t=1}^{N}\left[\frac{\mathrm{V}_{\mathrm{P}}-\mathrm{V}_{\mathrm{S}}}{\mathrm{V}_{\mathrm{S}}}\right]}{N} .
$$

In this relation, $N$ refers to the number of time frames in the event, $V_{\mathrm{P}}$ refers to the normalized velocity in the perturbed event, and $V_{\mathrm{S}}$ refers to the normalized velocity in the standard event. This measure will be referred to as mean velocity Weber fraction (MVW). This measure is not to meant to imply that participants make comparisons between normalized velocities at similar times in events separated by several seconds. However, this measure does allow comparison to typical velocity Weber fraction measures used in the motion perception literature. Because the MVW measure is not an event-specific measure beyond the requirement of equivalent time frames in the two events to be compared, it allows comparison between trajectory forms of arbitrary unidimensional events.

In Figure 3, frequencies of choosing the second display are shown plotted against corresponding MVW values. For this plot and analysis, the MVW values for perturbations in the first display were assigned negative values, and those for the perturbations in the second dis- play were positive. Because the dependent measure was dichotomous, we used a probit analysis to fit a cumulative normal curve to these data (Amemiya, 1981; Finney, 1971). Cumulative normals were fit to each participants' responses. The MVW at which the participants judged trials correctly $25 \%$ and $75 \%$ of the time were averaged to obtain the threshold measure for each individual participant. The median of these thresholds was used to rep-

Table 1

Thresholds and Chi-Square $\left(\chi^{2}\right)$ Values From Choice Data (Averages of $25 \%$ and $75 \%$ Levels) of Experiments 1-3

\begin{tabular}{|c|c|c|c|c|}
\hline Event & Feedback & Participant & Threshold & $\chi^{2}$ \\
\hline \multicolumn{5}{|c|}{ Experiment 1} \\
\hline \multicolumn{5}{|l|}{ Asymmetric } \\
\hline \multirow[t]{3}{*}{ Pendular } & \multirow[t]{3}{*}{ No } & Median & .119 & \\
\hline & & G.B. & .144 & 231.1 \\
\hline & & M.S. & .094 & 384.8 \\
\hline \multicolumn{5}{|c|}{ Experiment 2} \\
\hline \multirow{8}{*}{$\begin{array}{l}\text { Asymmetric } \\
\text { Straight Path }\end{array}$} & \multirow{6}{*}{ No } & & & \\
\hline & & Median & .075 & \\
\hline & & E.W. & .041 & 196.1 \\
\hline & & K.S. & .063 & 132.1 \\
\hline & & M.E. & .118 & 81.7 \\
\hline & & L.W. & .086 & 88.7 \\
\hline & \multirow[t]{2}{*}{ Yes } & E.W. & .022 & 164.4 \\
\hline & & K.S. & .067 & 73.1 \\
\hline \multicolumn{5}{|c|}{ Experiment 3} \\
\hline \multirow[t]{7}{*}{ Peaked Symmetric } & \multirow[t]{5}{*}{ No } & Median & .112 & \\
\hline & & E.W. & .111 & 245.2 \\
\hline & & K.S. & .117 & 227.0 \\
\hline & & M.E. & .112 & 248.2 \\
\hline & & M.F. & .107 & 236.8 \\
\hline & \multirow[t]{2}{*}{ Yes } & E.W. & .063 & 188.2 \\
\hline & & K.S. & .074 & 162.8 \\
\hline \multirow[t]{7}{*}{ Flattened Symmetric } & \multirow[t]{5}{*}{ No } & Median & .075 & \\
\hline & & E.W. & .061 & 211.0 \\
\hline & & K.S. & .081 & 171.3 \\
\hline & & M.E. & .107 & 185.7 \\
\hline & & M.F. & .068 & 87.9 \\
\hline & \multirow[t]{2}{*}{ Yes } & E.W. & .067 & 99.7 \\
\hline & & K.S. & .087 & 81.0 \\
\hline
\end{tabular}

Note-For all $\chi^{2}$ values, $p s<.0001$. 
resent the combined responses of all participants. As shown in Table 1 and Figure 3, the individual participants' MVWs at threshold were .144 (for G.B.) and .094 (for M.S.). The median MVW between participants of about $12 \%$ at threshold might be compared with a typical velocity Weber ratio of about $5 \%$. This $12 \%$ value represents a displacement of the peak velocity equal to about $14 \%$ of the amplitude of the oscillation or $0.314^{\circ}$ (or about $20^{\prime}$ of arc). Each of the participants' thresholds fell outside of the $95 \%$ confidence interval of the other. Although both participants were able to distinguish modest changes in trajectory form, M.S.'s performance was better. As an indication of the success of the fit of the probit parameters to the data, we computed chi-square $\left(\chi^{2}\right)$ goodness-of-fit values based on maximum-likelihood ratios. The $\chi^{2}$ values were $231.1(p<.001)$ for G.B. and $384.8(p<.001)$ for M.S. Finally, sign tests performed separately for each participant's catch trials showed that there were pair order biases in G.B.'s judgments $(N=99$, $p=.03)$ but not in M.S.'s judgments $(N=99, p=.16)$. G.B. tended to select the first of the two displays in a trial when both were standard events.

\section{EXPERIMENT 2 Isolating Trajectory Form via Amplitude Variation}

In Experiment 1, the circle followed the same path of motion in standard and perturbed displays, but with different velocity profiles along the path. Because both the path and the amplitude of motion were the same in each case, trajectory form variations were not isolated as the only source of information that could be used to discriminate the perturbations. The participants could have based judgments on differences in the absolute velocity values at given locations along the path, such as the midpoint of motion. Thus in Experiment 1, two types of visual information may have been used by the participants: absolute values of display kinematics or variations in the trajectory forms of the events. In Experiment 2, trajectory forms were isolated as information to be used in the discrimination task by varying the amplitude of motion between the standard and comparison displays shown in each trial and keeping the periods of the displays the same. This manipulation is equivalent to varying the viewing distance. It renders all of the corresponding absolute kinematic values different in the standard and comparison displays and, thus, eliminates the possible use of any absolute kinematic values in judgments. Variation in trajectory form is left as the sole source of information that could be used to discriminate the events.

Although no rod was visible in the displays of Experiment 1 , it remained possible that the participants detected angular and straight path motion in the displays. In Experiment 2, a straight path oscillation was used instead of a pendular oscillation. This simplified the displays, leaving only straight path motion in a frontoparallel plane.

\section{Method}

Participants. Four graduate students in the Indiana University Psychology Department participated in this experiment. The participants were compensated at $\$ 6$ per hour for their participation. All had normal or corrected-to-normal vision.

Display generation. The displays were the same as those in Experiment 1 , with the following exceptions. The participants saw a black circle move in a straight path side to side on a white background. Also, the amplitude of motion was varied between the standard and comparison displays. Periods of oscillation were maintained at $2.145 \mathrm{sec}$ for all displays. Instead of a pendulum, a harmonic oscillator was used to generate the motions.

Manipulation of the amplitude of each display was achieved by setting the simulated viewing distance to a different value before recording each display. The viewing distance was selected randomly from a range of far viewing distances for one display in a pair and a range of close viewing distances for the other display. The far viewing distances produced a range of displacements from $5.28^{\circ}$ to $9.24^{\circ}$ of visual angle. The closer viewing distances produced a range from $11.86^{\circ}$ to $15.77^{\circ}$ of visual angle. This resulted in average optical velocities ranging from $4.92^{\circ}$ to $14.7^{\circ}$ per second. These velocities fall within the range often studied in the motion perception literature and for which velocity difference thresholds are typically between 5\% and 7\% (McKee, 1981; Orban et al., 1984; Werkhoven et al., 1992). Which display was at a large amplitude was counterbalanced, but otherwise randomized, across standard and comparison displays and the first or second display viewed.

Design. The three perturbation levels were determined for each participant using that participant's performance in pilot experiments. Levels were chosen to correspond to below $75 \%$ (above 25\%) correct performance, $75 \%(25 \%)$ correct performance, and performance yielding near $100 \%(0 \%)$ correct performance. These three levels of perturbation were combined with two orderings (standardperturbed or perturbed -standard) to yield six pair order combinations. Each participant judged each of these six conditions 50 times, resulting in 300 trials. The displays were completely randomized and presented to each participant in two 150-trial sessions, lasting about $1 \mathrm{~h}$ each. The participants took brief breaks after every 30 trials.

Procedure. The procedure used was the same as that in Experiment 1 .

\section{Results}

Calculation of MVWs for the perturbed trajectory forms was the same as in Experiment 1. MVW ratios varied from .162 for the strongest perturbation to .019 for the weakest.

Cumulative normal functions were fit to responses of individual participants using probit analysis. As shown in Table 1 and Figure 4, the MVW ratios at threshold were as follows for each of the 4 participants: .041 (E.W.), .063 (K.S.), .118 (M.E.), and .086 (L.W.). The median of the participants' MVW ratios at threshold was .075. Chi-square values for the individual participants were as follows: 196.1 for E.W. $(p<.001), 132.1$ for K.S. $(p<.001), 81.7$ for M.E. $(p<.001)$, and 88.7 for L.W. $(p<.001)$. Additionally, sign tests performed on the participants' responses indicated that there were no pair order biases $(N=300, p=.386$, for E.W.; $N=300$, $p=.119$, for K.S.; $N=300, p=.326$, for M.E.; $N=300$, $p=.149$, for L.W.).

We assessed whether those thresholds were indicative of optimal performance by having 2 participants (E.W. 

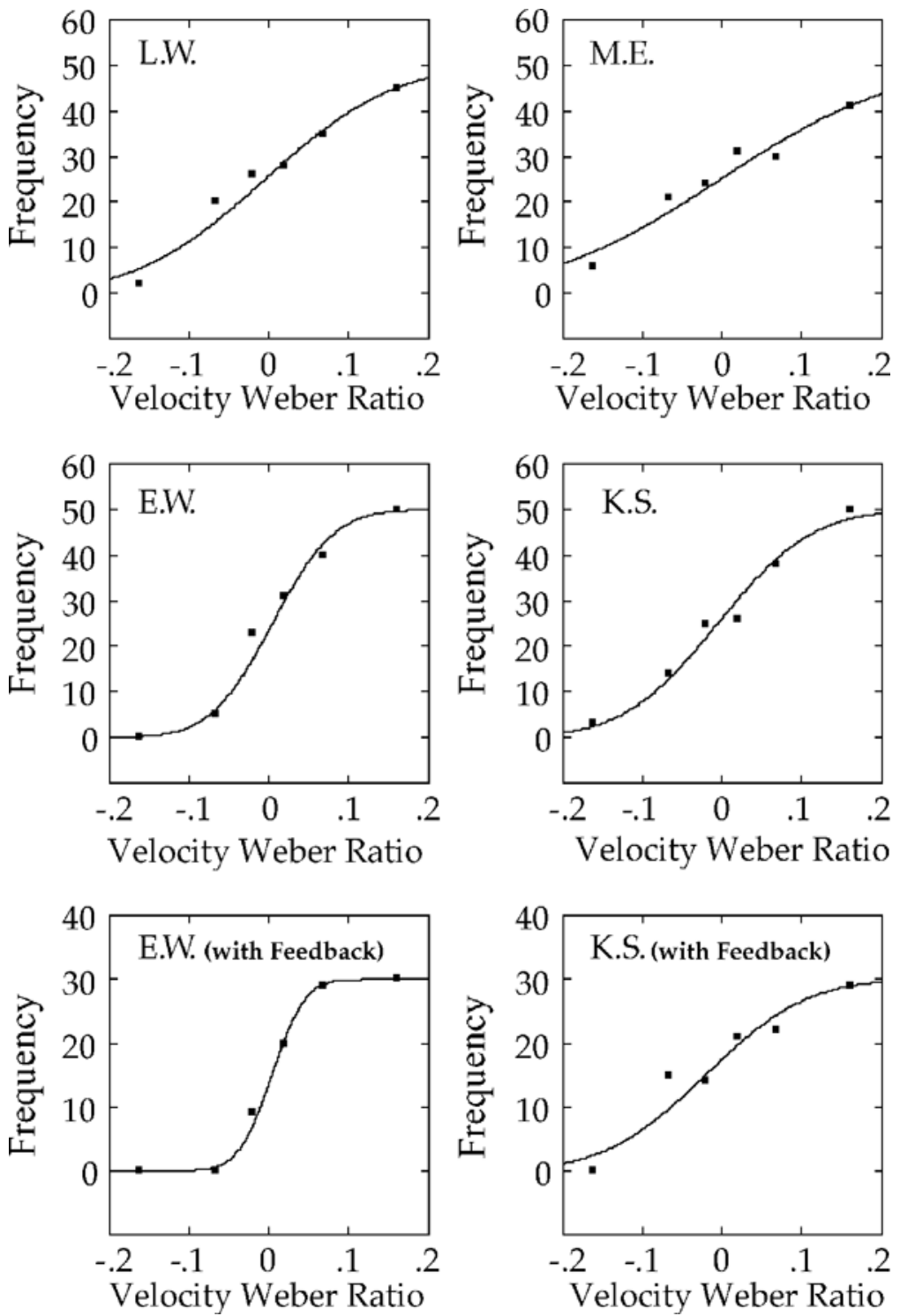

Figure 4. Cumulative normal curves fitted by probit analysis to the observed frequencies in selecting the second display as distorted. Curves and data plotted against mean velocity Weber ratios for Experiment 2.

and K.S.) complete a revision of Experiment 2, including feedback about which of the two displays was the perturbed event. The revision had 180 trials rather than 300 trials. Also, in an attempt to optimize performance, the participants were tested in two sessions composed of three blocks of trials of descending strengths of perturbation. The results are shown in Figure 4. The MVW ratios at threshold for E.W. and K.S. were $.022\left(\chi^{2}=164.4\right.$, $p<.001)$ and $.067\left(\chi^{2}=73.1, p<.001\right)$, respectively. Given the absence of a pair order bias, we used parallelism tests to evaluate the potential difference in thresholds. The result for E.W. was a $\chi^{2}$ of $7.14(p<.001)$. The drop of threshold from .041 to .022 was significant. The result for K.S. was a $\chi^{2}$ of $.25(p>.5)$. The threshold did not change (.063 vs. .067). So, feedback yielded improved performance in only 1 of the 2 participants tested.

These results showed clearly that participants are able to discriminate differences in trajectory forms. More than this, because the participants were required to select the perturbed display (rather than merely judge that a difference was present), the results indicate that participants are able to detect a trajectory form. Across participants, the MVW ratio at threshold was $7.5 \%$. (Note that this represents the mean amount of velocity difference at comparable times in trajectories. The maximum velocity Weber fraction comparing velocities occurring at similar 
times in the events at threshold was $11.6 \%$. Similarly, the maximum velocity Weber fraction comparing velocities across positions was $13.9 \%$.) This level of performance, $7.5 \%$, is comparable to that found for optical velocity thresholds $(\approx 5 \%)$, although no direct comparison of velocities between standard and comparison displays could have yielded these results because the absolute velocities were all different in any given standard and comparison pair at comparable times or positions within the events.

We compared the results of Experiment 1 with those of Experiment 2 using a parallelism test (although a pair order bias was found for 1 participant in Experiment 1). The $\chi^{2}$ was $44.23(p<.001)$. The difference was significant. The ratio of the overall MVW thresholds of Experiments 1 and 2 was equal to 2.32. This indicates that there had to be twice as much deviation in trajectory form for the pendular events in Experiment 1 to be detected as that for the straight path events in Experiment 2. There are two possible accounts for the improved performance in Experiment 2. First, the curved paths of movement in Experiment 1 may have reduced sensitivity to speed variations along the path of motion. Second, the availability of comparison in terms of absolute velocity differences in Experiment 1 may have inspired the participants to use this information, even though it led to inferior discrimination.

Whatever the reason for this difference in performance, the results of Experiment 2 clearly show that participants are able to discriminate events on the basis of subtle differences in trajectory forms, as seen in Figure 5. In particular, the participants were sensitive to the effects of nonlinear dissipative dynamical components on the form of an event.

\section{EXPERIMENT 3 \\ Symmetry-Preserving Perturbations in Trajectory Forms}

Having shown that participants are sensitive to perturbations that alter the symmetry of trajectory forms, we next investigated whether perturbations that preserved the symmetries would be detected. Dissipative forces were used to introduce asymmetric change in the harmonic form. Changes to the harmonic trajectory form that preserve its symmetries are produced by conservative forces (i.e., nonlinear springs). A hard spring produces a flattening of the circular form to yield a rounded rectangle. A soft spring produces a peakening of the circular form to yield a rounded diamond. Sensitivity to these changes, and, thus, sensitivity to effects of nonlinear conservative dynamic components, was tested in Experiment 3 .

\section{Method}

Participants. The same 4 participants as in Experiment 2 performed in this experiment.

Display generation. We systematically varied the curvature of an elliptical trajectory form while preserving the symmetry of the form and the period and amplitude of the oscillating event. Soft and hard springs were used to produce symmetric trajectory form per-

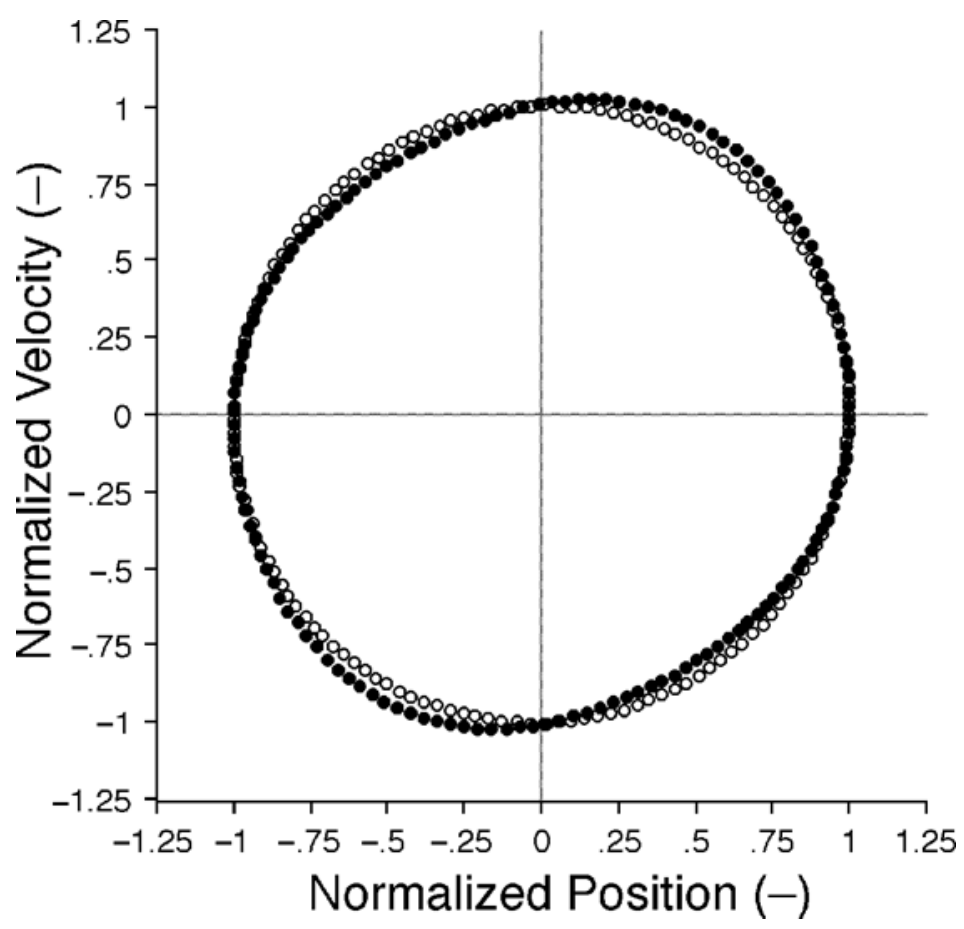

Figure 5. Threshold level of trajectory form variation in an asymmetric straight path oscillator (van der Pol) (filled circles) versus the standard symmetric straight path oscillator (harmonic) (unfilled circles). 


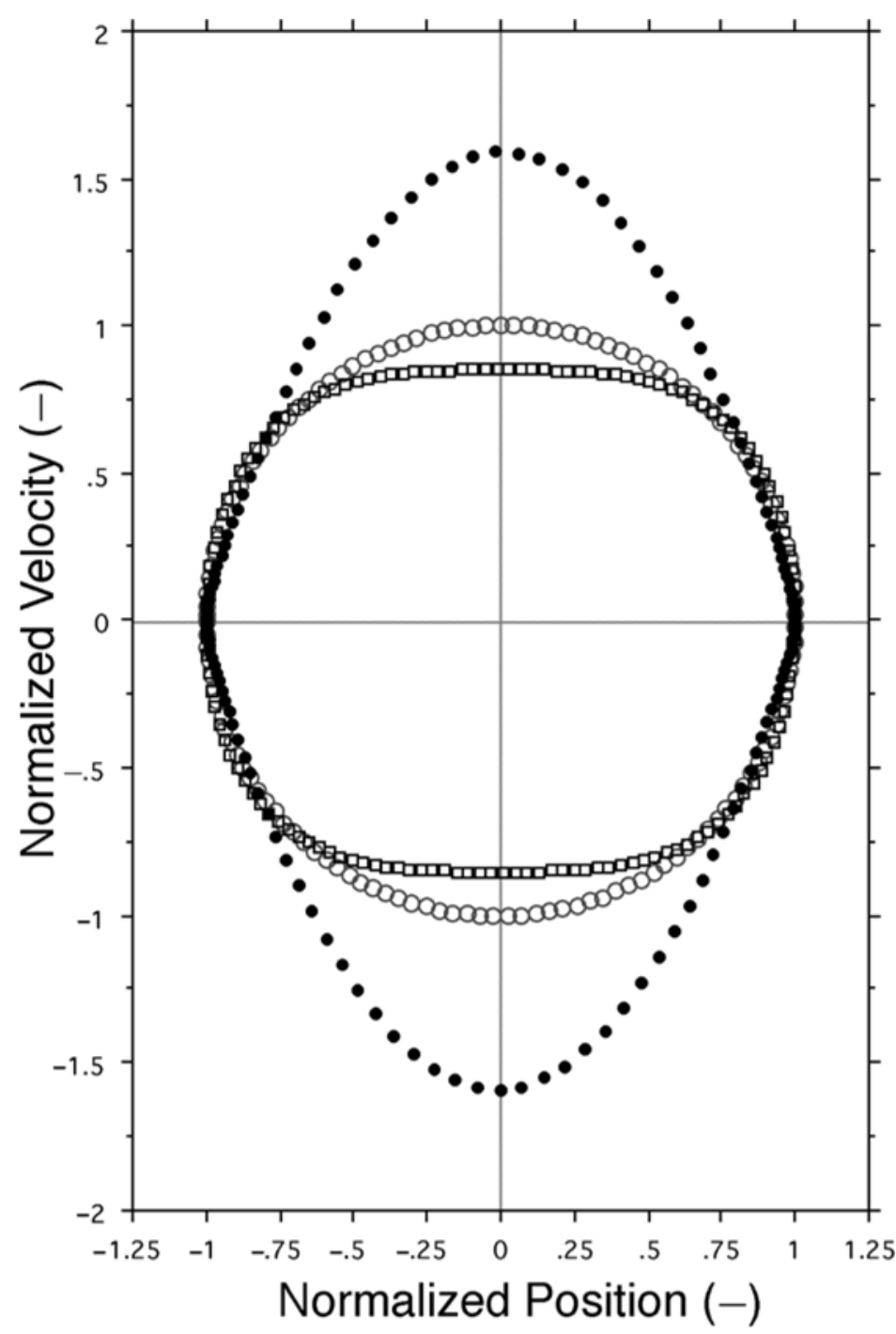

Figure 6. Trajectory form of a peaked symmetric straight path oscillator (soft spring) (filled circles), flattened symmetric straight path oscillator (hard spring) (unfilled squares), and symmetric straight path oscillator (harmonic) (unfilled circles).

turbations of the standard oscillator. The equation of motion of a soft spring was $\ddot{x}=-c\left(x-k x^{3}\right)$, where $c$ is a constant used to scale the period of the event, and $k$ is used to manipulate the peakedness of the trajectory form. The values of $c$ varied between 9.1 and 10.3, and $k$ varied between 0.013 and 0.019 . The comparable equation for the hard spring was $\ddot{x}=-c\left(x+k x^{3}\right)$. The values of $c$ varied between 3.66 and 0.49 , and $k$ varied from 1.83 to 2.95 . When $k=0$, the standard harmonic oscillator is produced. In the soft spring equation, as $k$ becomes increasingly large, the trajectory form becomes peaked, as shown in Figure 6. As $k$ becomes increasingly large in the hard spring equation, the trajectory form becomes flattened, as shown in Figure 6. The period of motion in all cases was $2 \mathrm{sec}$. All other characteristics of the displays were similar to those of Experiment 2. As in Experiment 2, the amplitudes of the standard and comparison displays were selected from distinct ranges simulating viewing from a range of near or far distances. The amplitude ranges were the same as those in Experiment 2.
Procedure. The design and procedure of this experiment were the same as those in Experiment 2, except for the following. The three levels of perturbation refer to the degree of peaking or flattening in the trajectory forms. The participants saw all of the peaked comparison pairs in two 162-trial sessions, for a total of $54 \times 6$ pairings $=324$ trials. Following this, the participants saw the flattened comparison pairs in two 162-trial sessions. Prior to each session, the participants were given a demonstration consisting of three trials, as in Experiment 2, but with either peaked or flattened events as the perturbed portion of the pair of displays.

\section{Results}

Calculation of MVW ratios was identical to that in Experiments 1 and 2. MVW ratios for the peaked events varied from .076 to .396 , and those for the flattened events varied from .061 to .135 . The range of values to 

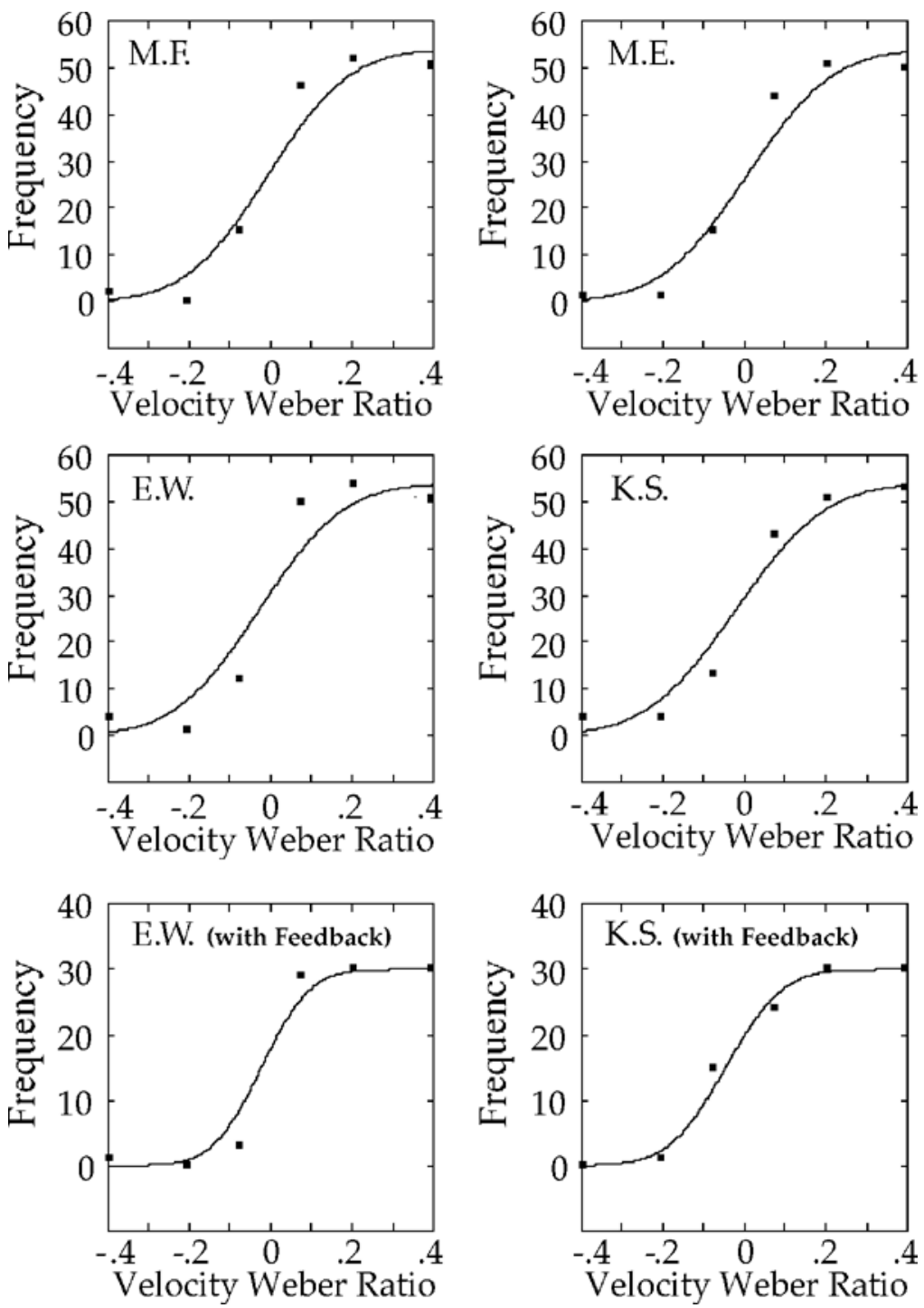

Figure 7. Cumulative normal curves fitted by probit analysis to the observed frequencies in selecting the second display as distorted. Curves and data plotted against mean velocity Weber ratios for symmetrical peaked events in Experiment 3.

be tested had been determined in pilot experiments, as in Experiment 2.

Cumulative normal curves were fit to each set of participant responses for each of the two types of trajectory form variation. As shown in Table 1 and Figures 7 and 8 , individual thresholds for the peaked events were .111 (E.W.), .117 (K.S.), .112 (M.E.), and .107 (M.F.). For the flattened events, the thresholds were .061 (E.W.), .081 (K.S.), .107 (M.E.), and .068 (M.F.). The median thresholds for the participants in each event were .112 for the peaked events and .075 for the flattened events. For the individual participants, the $\chi^{2}$ values for the peaked events were $245.2(p<.001)$ for E.W., $227.0(p<.001)$ for K.S., $248.2(p<.001)$ for M.E., and $236.8(p<.001)$ for M.F.; the values for the flattened events were 211.0 $(p<.001)$ for E.W., $171.3(p<.001)$ for K.S., $185.7(p<$ $.001)$ for M.F., and $87.9(p<.001)$ for M.E. Figure 9 shows the trajectory forms for the peaked and flattened symmetric events at the threshold for the combined participants.

Sign tests performed on the participants' responses indicated that there was no pair order bias in their judgments of either the peaked events $(N=324, p=.291$, for E.W.; $N=324, p=.541$, for K.S.; $N=324, p=.697$, for M.F.; $N=324, p=.999$, for M.E.) or the flattened events $(N=324, p=.868$, for E.W.; $N=324, p=.541$, for K.S.; $N=324, p=.781$, for M.F.; $N=324, p=.107$, for M.E.).

As in Experiment 2, we tested whether the participants' thresholds were indicative of optimal performance. Similar revisions of Experiment 3 (180 trials 

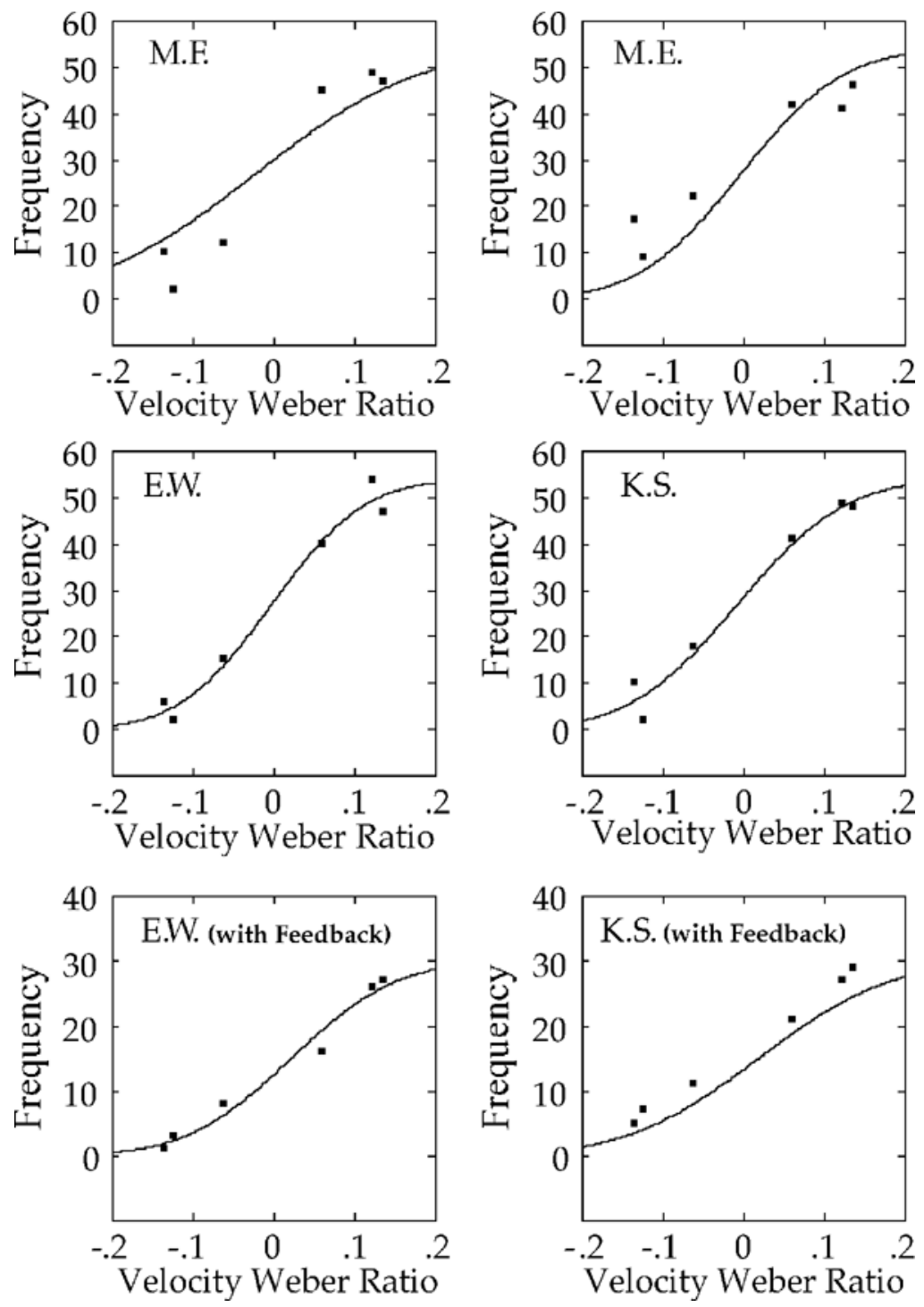

Figure 8. Cumulative normal curves fitted by probit analysis to the observed frequencies in selecting the second display as distorted. Curves and data plotted against mean velocity Weber ratios for symmetrical flattened events in Experiment 3.

rather than 324 trials) were performed, with feedback indicating which display contained the perturbed event. Also, in an attempt to maximize performance for each of the revisions, the participants were tested in two sessions composed of three blocks of trials of descending strengths of perturbation. Again, 2 participants were tested. For the peaked events, the thresholds for the revised experiment were $.063\left(\chi^{2}=188.2, p<.001\right)$ and $.074\left(\chi^{2}=\right.$ $162.8, p<.001)$ for E.W. and K.S., respectively. For the flattened events, their thresholds were $.067\left(\chi^{2}=99.7\right.$, $p<.001)$ and $.087\left(\chi^{2}=81.0, p<.001\right)$, respectively. Feedback appears to have improved performance in discriminating the peaked events, but not the flattened events. Again, given the absence of pair order biases, we used parallelism tests to test differences between thresh- olds. For the peaked events, the result was not significant for E.W. $\left(\chi^{2}<0.001, p=1\right)$ but was significant for K.S. $\left(\chi^{2}=19.1, p<.001\right)$. Because E.W. was performing at or near ceiling, this result should be treated with caution. Her performance did seem to improve with feedback, as that of K.S. certainly did. Thresholds dropped from $11 \%$ to $6.8 \%$. For flattened events, the test failed to reach significance for either participant (E.W., $\chi^{2}<0.001$, $p=1$; K.S., $\chi^{2}=0.17, p>.5$ ). Thresholds remained at about $7 \%$.

We used a parallelism test to compare the peaked and flattened events using the performance of the four participants without feedback. The result was significant $\left(\chi^{2}=323.05, p<.001\right)$. Thresholds were about $4 \%$ lower for the flattened events than for either the peaked events 


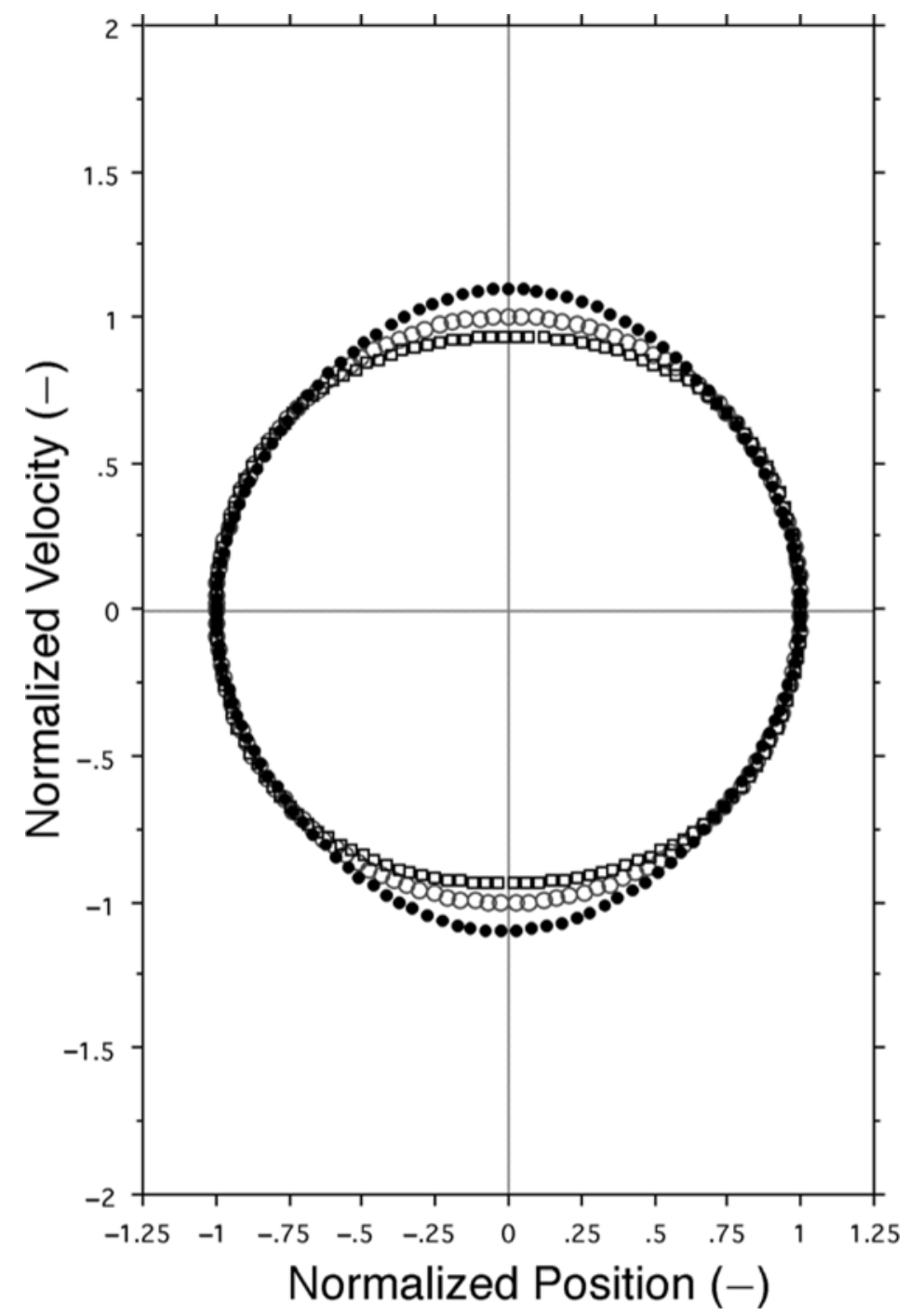

Figure 9. Threshold level of trajectory form variation in a peaked symmetric straight path oscillator (filled circles) versus the standard symmetric straight path oscillator (harmonic) (unfilled circles). Threshold level of trajectory form variation in a flattened symmetric straight path oscillator (unfilled squares) versus the standard symmetric straight path oscillator (harmonic) (unfilled circles).

or the asymmetric events. The comparison of peaked events with asymmetric events was similarly significant $\left(\chi^{2}=247.26, p<.001\right)$, but the comparison of flattened events with asymmetric events was not significant $\left(\chi^{2}=\right.$ $1.80, p>.15)$. With feedback, performance levels became strictly comparable at about $7 \%$.

\section{EXPERIMENT 4}

\section{Event Recognition Using Trajectory Forms}

If participants can use trajectory form information to discriminate events, could they also use this type of information to recognize particular events? In other words, could participants recognize a particular event when the only information about its identity is contained in the trajectory form?

The previous experiments demonstrated that participants can discriminate changes in trajectory forms, but the results provided evidence that could only suggest that trajectory forms could be used to recognize events. The participants did have to select the perturbed events, but they were tested only with a single type of perturbation at a time. As a test of whether participants can recognize events based on their trajectory forms, we asked the participants to identify a single viewed event from a set of possible events in Experiment 4. 
Table 2

Confusion Matrix for Events Presented in Experiment 4

\begin{tabular}{lcccc}
\hline & \multicolumn{4}{c}{ Judgments } \\
\cline { 2 - 5 } \multicolumn{1}{c}{ Event } & Harmonic & Asymmetric & Peaked & Flattened \\
\hline Harmonic & 60 & 8 & 13 & 19 \\
Asymmetric & 10 & 60 & 19 & 10 \\
Peaked & 11 & 20 & 64 & 5 \\
Flattened & 29 & 10 & 7 & 53 \\
\hline
\end{tabular}

Note-All values are percentages.

\section{Method}

Participants. Four graduate students from the Indiana University Psychology Department participated in this experiment. Two participants (E.W. and K.S.) had participated in Experiments 2 and 3 and were familiar with the events. The other 2 participants (D.M. and N.B.) did not have prior experience with these events, although D.M. was familiar with the rationale for these experiments. The participants were compensated $\$ 6$ per hour for their participation. All had normal or corrected-to-normal vision.

Display generation. Four types of event displays were used in this experiment. The simple harmonic oscillator used as the standard in the previous experiments was one type of event. The other types were taken from the perturbed event types used in Experiments 2 and 3: an asymmetric event, a peaked symmetric event, and a flattened symmetric straight path oscillator. Unlike in Experiments 2 and 3, amplitude variations were not used in Experiment 4. Rather, the amplitudes of the events were all equal. The visual angles subtended by all four events in Experiment 4 were $10.54^{\circ}$ of visual angle. Periods were $2 \mathrm{sec}$ for all four events, resulting in an average optical velocity of $10.54^{\circ}$ per second. The amount of perturbation in the latter three event types was that necessary for the participants to, on average, correctly distinguish each of these events from the harmonic oscillator at an $80 \%$ correct performance level.

Procedure. The participants performed a categorization task. During each of 99 trials, each participant was shown one of the four types of events and then asked to identify which of the four events was displayed. They were given feedback after each trial indicating which of the four types of events had been shown. Prior to the session, the participants were allowed to view samples of each of the types of events. They were free to view Events A, B, C, and D as many times they judged necessary to become familiar with the four types of events. Event A was the harmonic event, Event B was the asymmetric event, Event $\mathrm{C}$ was the peaked symmetric event, and Event $\mathrm{D}$ was the flattened symmetric event. The order in which the participants viewed the four events was not restricted during this familiarization procedure.

\section{Results}

Chi-square tests indicated that all of the participants performed above chance in correctly identifying each of the types of events. Separate tests were performed for each participant [E.W., $\chi^{2}(9)=217.74, p<.001$; K.S., $\chi^{2}(9)=350.20, p<.001$; D.M., $\chi^{2}(9)=222.53, p<.001$; N.B., $\left.\chi^{2}(9)=373.17, p<.001\right]$ and for all participants combined $\left[\chi^{2}(9)=1,065.82, p<.001\right]$. The participants were well above chance performance $(25 \%)$ in correctly identifying each event $(60 \%, 60 \%, 64 \%$, and $53 \%$, for the harmonic, asymmetric, peaked symmetric, and flattened symmetric events, respectively, for the combined participants). However, they tended to confuse the harmonic and flattened symmetric events with each other and confuse the asymmetric and peaked symmetric events with each other. Table 2 shows this pattern of confusions for the combined responses of all of the participants.

\section{GENERAL DISCUSSION}

We sought to test whether participants can make use of trajectory forms to recognize events, as well as the salience of types of perturbation to trajectory forms, including asymmetry and symmetrical peakening or flattening in a trajectory form. Sensitivity to asymmetrical changes in trajectory forms is potentially important, because these changes can be indicative of biological movements, such as studied by Bingham et al. (1995). Symmetrical peakening or flattening of trajectory forms are likewise potentially important. Bingham(1987) found that the flattening of an otherwise round harmonic form provided information to observers that the amount of weight being lifted by a person was near to the maximum liftable. Also, because asymmetry entails a change in the peakedness of a form, studying symmetrical peakening helps to differentiate the relative sensitivities to asymmetrical versus peakedness variations of trajectory forms.

We found that the participants were able to use variations in trajectory forms as visual information about events. We isolated trajectory forms as information in Experiments 2 and 3 by holding the period of oscillatory events constant while varying the amplitude of the two events to be compared. First, we found that the participants were able to distinguish events that differed only in their trajectory forms. The participants distinguished an asymmetric form from the symmetric trajectory form of a harmonic oscillator. Also, they distinguished symmetric peakening or flattening of the harmonic form. Without feedback, the participants were slightly less sensitive to the peakening of the harmonic form than to the flattening or the asymmetric change. The thresholds were $11.2 \%, 7.5 \%$, and $7.5 \%$, respectively. With feedback, these dropped slightly to $6.8 \%, 7.7 \%$, and $4.4 \%$, respectively. Second, in Experiment 4, we found that distinct trajectory forms allowed participants to identify specific events. Given near threshold differences, the participants reliably identified the four types of events: circular (or harmonic or linear spring), asymmetric (or van der Pol), peaked symmetric (or soft spring), and flattened symmetric (or hard spring).

We have discovered that participants can use trajectory form information to discriminate and identify events. This raises the following question: What was the specific optical property used to detect the trajectory forms? In fact, our measure of the relative threshold was provisional. We chose the MVW ratio as an index of the degree of form change in these events because it could be applied equally well to each of the three types of perturbations to the harmonic trajectory form. Also, the measure allowed rough comparison to mean Weber ratios from velocity perception studies. Many studies demonstrate direct detection of velocity by the visual system (Brown, 1961; Lappin, Bell, Harm, \& Kottas, 
Table 3

Comparison of Thresholds for Trajectory Form Measures

\begin{tabular}{clcccc}
\hline \multirow{5}{*}{ Event } & & Temporal & & \multicolumn{3}{c}{$\begin{array}{c}\text { Velocity } \\
\text { Spatial } \\
\text { Variance } \\
\text { MVW } \\
\text { Ratio }\end{array}$} & $\begin{array}{c}\text { Weber } \\
\text { Ratio }\end{array}$ & $\begin{array}{c}\text { Curvature } \\
\text { Reber } \\
\text { Ratio }\end{array}$ \\
\hline Asymmetric & All & .075 & .068 & .008 & .218 \\
& E.W. & .041 & .042 & .005 & .144 \\
& K.S. & .063 & .059 & .007 & .194 \\
& M.E. & .118 & .082 & .010 & .300 \\
& L.W. & .086 & .077 & .009 & .235 \\
Symmetric & & & & & \\
& & & & & \\
& All & .112 & .103 & .103 & .203 \\
& E.W. & .111 & .102 & .104 & .185 \\
& K.S. & .117 & .106 & .108 & .232 \\
& M.E. & .112 & .103 & .101 & .184 \\
& M.F. & .107 & .098 & .097 & .210 \\
Flattened & All & .075 & .045 & .036 & .202 \\
& E.W. & .061 & .040 & .032 & .120 \\
& K.S. & .081 & .046 & .037 & .206 \\
& M.E. & .107 & .070 & .056 & .304 \\
& M.F. & .068 & .044 & .035 & .176 \\
\hline
\end{tabular}

Note-MVW, mean velocity Weber.

1975; McKee, 1981; Orban et al., 1984) at a Weber Fraction of 5\% (McKee, 1981; Nakayama, 1981; Orban et al., 1984; van Doorn \& Koenderink, 1983). As shown in Table 3, in these terms, our results were certainly comparable - that is, MVW ratios at threshold $(\approx 7 \%)$ were comparable to the $5 \%$ value representative of velocity difference thresholds.

Velocities might have been compared as a function of comparable positions rather than comparable times. Events necessarily occur over time; however, they also occur over spatial layouts. For example, a bouncing ball, a hopping frog, or a walking person all progress over a ground surface. Events in general unfold against a background of surfaces, a spatial layout. Bingham (1995) was partly inspired by this observation to characterize events as spatiotemporal objects possessing spatiotemporal forms - that is, trajectory forms. Accordingly, velocity variations in events may well be detected in a spatial frame of reference. If this is so, then it would be better to assess performances on a spatial scale rather than a temporal scale. So, we computed MVW measures at equal proportionate spatial positions, rather than equal time intervals, comparing the perturbed and standard events in Experiments 2 and 3. Table 3 shows the thresholds for this spatial MVW measure, together with those for the original temporal MVW measure. Both the essential values and the pattern of the thresholds across the events was similar when computed using either temporal or spatial frames of reference. ${ }^{2}$

However, given that the absolute velocities in Experiments 2 and 3 were not the same at any of the corresponding locations or times within the events, even when the trajectory forms were the same, the participants cannot have discriminated the forms in terms of direct velocity comparisons. Use of proportionate velocities at proportionate locations would be a way to detect and discriminate differences in trajectory forms, but it is unlikely that participants do this, and, if they did, we would not expect them to exhibit comparable levels of sensitivity to both absolute velocity differences and differences of such transformed or normalized velocities. Yet, their level of performance reflects comparable sensitivities on these measures. Also, if the same velocity measure were being used in each of these different comparisons, then we would expect to see the same threshold values. While the values are close, they are nevertheless about half again as large for the peaked event as for the asymmetric or flattened. These observations imply that participants must be using a different measure. So, what might they be using?

Another possibility is that differences in trajectory forms were detected as differences in the distribution of velocities along a path of motion. Werkhoven et al. (1992) proposed that accelerations might be detected via a velocity variance mechanism. We computed a velocity variance Weber ratio using the harmonic motion as the standard, just as we had in computing the MVW measure, and then we computed thresholds using this measure with probit analysis. Analysis of the results from Experiments 2 and 3 showed that the participants would had to have detected minute changes in the variance of the velocities between standard and comparison displays, as shown in Table 3. For instance, to discriminate the asymmetric from the harmonic trajectory form at threshold, the participants would had to have detected a difference of $0.8 \%$. This is well below the $3 \%$ threshold found by Werkhoven et al. for this measure. Under the assumption that the participants were using only a single measure to distinguish and identify all of these trajectory forms, then, they could not have been using a velocity variance approach.

It is possible nevertheless that the participants may have used two measures. They may have used velocity variance to detect changes in trajectory form that preserved the spatial symmetry and then another separate measure to detect asymmetry. But, if this were the case, then we would expect the thresholds for the two symmetric variations, peaked and flattened, to be the same, and they were not. The VVW threshold for the peaked event $(10.3 \%)$ was nearly three times that for the flattened event $(3.6 \%)$. So, it is unlikely that a velocity variance measure was used.

A logical possibility in the detection of trajectory forms would be some measure that is computed as a function of acceleration. Acceleration could be detected directly (Rosenbaum, 1975) or arrived at indirectly through comparison of an earlier and later velocity (Gottsdanker, Frick, \& Lockard, 1961; Regan, Kaufman, \& Lincoln, 1986; Schmerler, 1976). Either way, however, observers have typically been found to be relatively insensitive to acceleration (Brown, 1961; Gottsdanker, 1956; Schmerler, 1976). Nevertheless, sensitivity to acceleration need not be required for an ability to detect variation in veloc- 

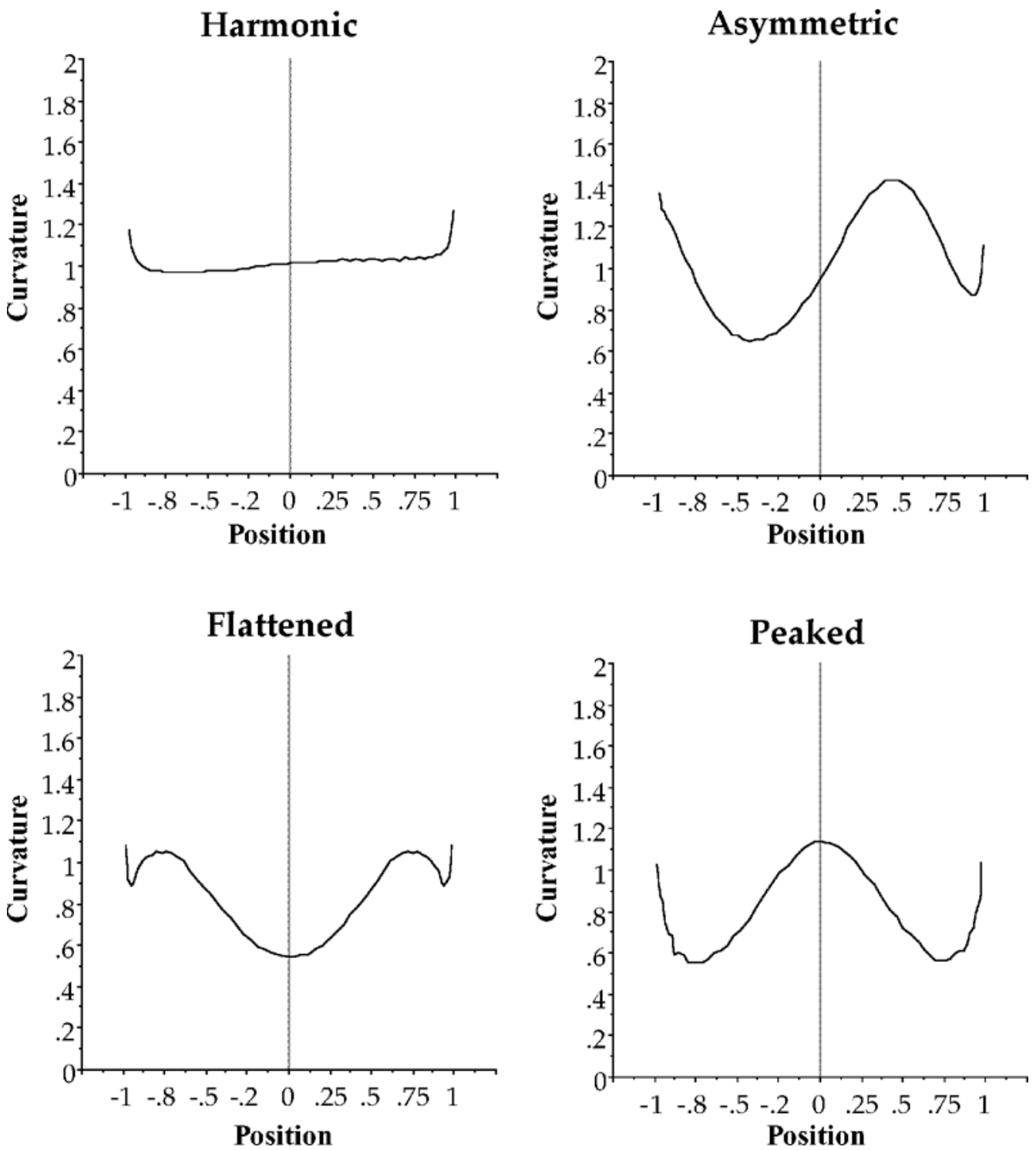

Figure 10. Plots of position versus the continuous curvature for the first half cycle of the four types of trajectory form.

ity along a trajectory. Acceleration is a measure of change in velocity over time - that is, $d v / d t$. As we have already observed, we should also consider changes in velocity over space, $d v / d x$. Following this observation, we next took our lead from studies of structure-from-motion, where investigators have also been searching for a visual measure of shape.

Studies of structure-from-motion have demonstrated that only first-order temporal properties are used in the perception of object shape from optic flow. Accelerations - that is, second-order temporal properties-are not used (Liter, Braunstein, \& Hoffman, 1993; Todd \& Bressan, 1990; Todd \& Norman, 1991). Nevertheless, higher order properties of motion are involved: spatial variations in velocity (i.e., $d v / d x, d^{2} v / d x^{2}$ ) and proper- ties derived from these (Perotti, Todd, Lappin, \& Phillips, 1998; see also Dijkstra, Snoeren, \& Gielen, 1994, and Koenderink \& van Doorn, 1992).

In structure-from-motion displays, a spatial distribution of optical velocities is available at a given moment in time. In events, of course, a spatial variation in velocity would be available over successive times. Nevertheless, variation in velocity along a path of motion detected in terms of spatial derivatives could yield sensitivity to trajectory forms in events. Such spatial variations in velocity correspond to the forms of trajectories plotted in phase space.

A quantity that has been studied in the structure-frommotion literature is curvature (Koenderink, 1990; Perotti et al., 1998; Tittle \& Perotti, 1997). There, it has been used 
to describe the structure of perceived surfaces. In the context of events, curvature might be a potential source of information about trajectory forms. That is, curvature might describe a property of trajectories that, if detected, would allow an event to be recognized. Curvature varies with the change in the slope along the trajectory in phase space. Curvature is computed using spatial derivatives of the velocities in an event, so it does not entail the general problem of insensitivity to accelerations. The curvature at each point along a trajectory is computed as follows:

$$
\text { curvature }(\kappa)=\frac{\left|d^{2} y / d x^{2}\right|}{\left[1+(d y / d x)^{2} \mid\right.},
$$

where $x$ is the position and $y$ is the velocity. We computed the curvature at each point along each of the trajectories used in Experiments 2 and 3. Then, we computed mean curvature Weber ratios for each of the comparisons in the same way that we had computed the MVW ratios. However, because the curvature of the standard harmonic trajectory is constant and equal to 1 , the mean curvature Weber ratios are equal to the mean absolute differences in curvature. We used these values in probit analyses to derive thresholds from the results of Experiments 2 and 3 . Table 3 shows thresholds for the mean curvature Weber ratio measure. The results show that a fairly substantial change in curvature is required to reach threshold, which is at about 20\%. (Unfortunately, there are no studies of curvature thresholds in the literature with which to compare this result.) However, we found that this index of sensitivity to changes in trajectory forms was the same for each of our three comparisons. This is exactly what we would expect for the appropriate measure. We explored a number of other potential measures, including the first and second spatial derivatives $\left(d v / d x\right.$ and $d^{2} v / d x^{2}$, respectively) and their ratio, but none of them exhibited patterns close to that required-that is, the thresholds for the different events were strongly different.

Figure 10 shows the continuous curvatures for each of the four types of events. Those for the asymmetric, flattened, and peaked events represent forms near threshold. It is, of course, the qualitative properties of these curvature functions that represent the forms. The harmonic is flat and exhibits no extrema. The peaked exhibits a single maximum centered between two minima. The flattened exhibits a single minimum centered between two maxima. The asymmetric exhibits a single maximum and a single minimum. (The computation yields small artifactual upturned tails on either end of these functions.) These qualitative features remain invariant over changes in the scale of the movement, and so it is these features that represent the trajectory forms as such. This single measure allows the four forms to be discriminated and identified. A second dimension is not required.

These portraits also relate to the pattern of confusions found in Experiment 4 and shown in Table 2. Asymmetric and peaked trajectory forms were often confused with one another, and harmonic and flattened forms were sim- ilarly confused. Both asymmetric and peaked trajectories exhibit a minimum on the left followed by a maximum to the right. In the peaked trajectory, the maximum is followed by another minimum farther to the right. This minimum is absent in the asymmetric trajectory, and this difference allowed the two trajectory forms to be discriminated most of the time. The flattened profile is well distinguished from either the peaked profile or the asymmetric profile because it has a minimum where they have a maximum, and vice versa. Apparently, the minimum in the flattened profile is more difficult to distinguish from the featureless harmonic than are the maxima in the other two profiles.

The curvature measure of trajectory forms should be verified in future studies. Nevertheless, the clear conclusion from this study is that human observers can detect trajectory forms and use them to distinguish and to recognize events. Events are spatiotemporal objects of perception that are recognized by virtue of their forms, just as are the more familiar spatial objects.

\section{REFERENCES}

AmemiY A, T. (1981). Qualitative response models: A survey. Journal of Economic Literature, 19, 1483-1536.

BInGHAm, G. P. (1987). Kinematic form and scaling: Further investigations on the visual perception of lifted weight. Journal of Experimental Psychology: Human Perception \& Performance, 13, 155-177.

BINGHAM, G. P. (1995). Dynamics and the problem of visual event recognition. In T. R. Port \& T. Van Gelder (Eds.), Mind as motion: Dynamics, behavior and cognition (pp. 403-448). Cambridge, MA: MIT Press.

Bingham, G. P., Rosenblum, L. D., \& Schmidt, R. C. (1995). Dynamics and the orientation of kinematic forms in visual event recognition. Journal of Experimental Psychology: Human Perception \& Performance, 21, 1473-1493.

Bingham, G. P., \& Runeson, S. (1983, October). On describing what is perceived: Seeing "velocity" versus seeing "push" in moving objects. Paper presented at a meeting of the International Society for Ecological Psychology, Trinity College, Hartford, CT.

Braunstein, M. L. (1976). Depth perception through motion. New York: Academic Press.

Braunstein, M. L. (1994). Structure from motion. In A. T. Smith \& R. J. Snowden (Eds.), Visual detection of motion (pp. 367-393). San Diego: Academic Press.

Brown, R. H. (1961). Visual sensitivity to differences in velocity. Psychological Bulletin, 58, 89-103.

Dijkstra, T. M. H., Snoeren, P. R., \& Gielen, C. C. A. M. (1994). Extraction of three-dimensional shape from optic flow: A geometric approach. Journal of the Optical Society of America A, 11, 2184-2196.

FInNeY, D. J. (1971). Probit analysis. Cambridge: Cambridge University Press.

GotTSDANKer, R. M. (1956). The ability of human operators to detect acceleration of target motion. Psychological Bulletin, 53, 477-487.

Gottsdanker, R. M., Frick, J. W., \& Lockard, R. B. (1961). Identifying the acceleration of visual targets. British Journal of Psychology, 52, 31-42.

Hoffman, D., \& Flinchbaugh, B. (1982). The interpretation of biological motion. Biological Cybernetics, 42, 197-204.

JAnsson, G., Bergst RÖM, S. S., \& EpsteIn, W. (EDS.) (1994). Perceiving events and objects. Hillsdale, $\mathrm{NJ}$ : Erlbaum.

Johansson, G. (1950). Configurations in event perception. Uppsala: Almqvist \& Wiksell.

JoHANSSON, G. (1973). Visual perception of biological motion and a model for its analysis. Perception \& Psychophysics, 14, 201-211.

JoHANSSON, G. (1976). Spatio-temporal differentiation and integration in visual motion perception. Psychological Research, 38, 279-293. 
Johansson, G., von Hofsten, C., \& Jansson, G. (1980). Event perception. Annual Review of Psychology, 31, 27-63.

Kay, B. A., Saltzman, E. L., \& Kelso, J. A. S. (1991). Steady state and perturbed rhythmical movements: A dynamical analysis. Journal of Experimental Psychology: Human Perception \& Performance, 17, 183-197.

Koenderink, J. J. (1990). Solid shape. Cambridge, MA: MIT Press.

Koenderink, J. J., \& VAN Doorn, A. J. (1992). Second-order optic flow. Journal of the Optical Society of America A, 9, 530-538.

LAPPIN, J. S. (1995). Visible information about structure from motion. In W. Epstein \& S. Rogers (Eds.), Perception of space and motion (pp. 165-199). New York: Academic Press.

Lappin, J. S., Bell, H. H., Harm, O. J., \& Kottas, B. (1975). On the relation between time and space in the visual discrimination of velocity. Journal of Experimental Psychology: Human Perception \& Performance, 1, 383-394.

Liter, J. C., Braunstein, M. L., \& Hoffman, D. D. (1993). Inferring structure from motion in two-view and multiview displays. Perception, 22, 1441-1465.

McKee, S. P. (1981). A local mechanism for differential velocity detection. Vision Research, 21, 491-500.

NakaYama, K. (1981). Differential motion hyperacuity under conditions of common image motion. Vision Research, 21, 1475-1482.

Norman, J. F., \& ToDD, J. T. (1993). The perceptual analysis of structure from motion for rotating objects undergoing affine stretching transformations. Perception \& Psychophysics, 53, 279-291.

Orban, G. A., DE Wolf, J., \& MAEs, H. (1984). Factors influencing velocity coding in the human visual system. Vision Research, 24, 33-39.

Perotti, V. J., Todd, J. T., Lappin, J. S., \& Phillips, F. (1998). The perception of surface curvature from optical motion. Perception \& Psychophysics, 60, 377-388.

Perotti, V. J., Todd, J. T., \& Norman, J. F. (1996). The visual perception of rigid motion from constant flow fields. Perception \& Psychophysics, 58, 666-679.

Regan, D. M., Kaufman, L., \& Lincoln, J. (1986). Motion in depth and visual acceleration. In K. R. Boff, L. Kaufman, \& J. P. Thomas (Eds.), Handbook of perception and human performance: Vol. 1. Sensory processes and perception (pp. 19-1 to 19-46). New York: Wiley.

Rosenbaum, D. A. (1975). Perception and extrapolation of velocity and acceleration. Journal of Experimental Psychology: Human Perception \& Performance, 1, 395-403.

Rosenberg, R. M. (1977). Analytical dynamics of discrete systems. New York: Plenum.

Runeson, S. (1974). Constant velocity-Not perceived as such. Psychological Research, 37, 3-23.

Schmerler, J. (1976). The visual perception of accelerated motion. Perception, 5, 167-185.

Thompson, J. M. T., \& STEWART, H. B. (1986). Nonlinear dynamics and chaos: Geometrical methods for engineers and scientists. New York: Wiley.
Tittle, J. S., \& Perotti, V. J. (1997). The perception of shape and curvedness from binocular stereopsis and structure from motion. Perception \& Psychophysics, 59, 1167-1179.

ToDD, J. T. (1982). Visual information about rigid and nonrigid motion: A geometric analysis. Journal of Experimental Psychology: Human Perception \& Performance, 8, 238-251.

TodD, J. T. (1983). Perception of gait. Journal of Experimental Psychology: Human Perception \& Performance, 9, 31-42.

ToDD, J. T. (1995). The visual perception of three-dimensional structure from motion. In W. Epstein \& S. Rogers (Eds.), Perception of space and motion (pp. 201-226). New York: Academic Press.

TodD, J. T., \& BRESSAN, P. (1990). The perception of 3-dimensional affine structure from minimal apparent motion sequences. Perception \& Psychophysics, 48, 419-430.

TodD, J. T., \& Norman, J. F. (1991). The visual perception of smoothly curved surfaces from minimal apparent motion sequences. Perception \& Psychophysics, 50, 509-523.

Todd, J. T., Tittle, J. S., \& Norman, J. F. (1995). Distortions of threedimensional space in the perceptual analysis of motion and stereo. Perception, 24, 75-86.

van Doorn, A. J., \& Koenderink, J. J. (1983). Detectability of velocity gradients in moving random dot patterns. Vision Research, 23, 799-804.

Werkhoven, P., Snippe, H. P., \& Toet, A. (1992). Visual processing of optic acceleration. Vision Research, 32, 2313-2329.

\section{NOTES}

1. We effectively treat frequency and amplitudes as fixed parameters. The effect on event perception of variations in these parameters is left for subsequent studies.

2. We should note, however, that this parity of spatial and temporal results cannot be expected in all cases. What may well distinguish a hopping frog from a bouncing ball is a brief elapse of time when velocity is 0 and the frog is in contact with the ground. This delay would be absent in the bouncing ball (see Bingham et al., 1995), where this difference may have contributed to the ability of observers to distinguish animate from inanimate bouncing motion. If this were the only difference in the trajectories of the frog and the ball, then it would remain invisible in the phase portrait where the trajectory simply goes into and comes out of the position axis at velocity equal to 0 . This means that a general treatment of trajectory forms might be expected to require something comparable to a state-time space (Rosenberg, 1977) with spatial, temporal, and velocity axes.

(Manuscript received December 30, 1999; revision accepted for publication March 7, 2001.) 